\title{
Yeast-based assay identifies novel Shh/Gli target genes in vertebrate development
}

\author{
Luis A Milla ${ }^{1,3 \dagger}$, Claudio R Cortés ${ }^{1,3 \dagger}$, Christian Hodar Q Q,3, Maritza G Oñate ${ }^{1,3}$, Veronica Cambiazo ${ }^{2,3}$, \\ Shawn M Burgess ${ }^{4}$ and Verónica Palma ${ }^{1,3^{*}}$
}

\begin{abstract}
Background: The increasing number of developmental events and molecular mechanisms associated with the Hedgehog $(\mathrm{Hh})$ pathway from Drosophila to vertebrates, suggest that gene regulation is crucial for diverse cellular responses, including target genes not yet described. Although several high-throughput, genome-wide approaches have yielded information at the genomic, transcriptional and proteomic levels, the specificity of Gli binding sites related to direct target gene activation still remain elusive. This study aims to identify novel putative targets of Gli transcription factors through a protein-DNA binding assay using yeast, and validating a subset of targets both invitro and in-vivo. Testing in different Hh/Gli gain- and loss-of-function scenarios we here identified known (e.g., ptc1) and novel Hh-regulated genes in zebrafish embryos.

Results: The combined yeast-based screening and MEME/MAST analysis were able to predict Gli transcription factor binding sites, and position mapping of these sequences upstream or in the first intron of promoters served to identify new putative target genes of Gli regulation. These candidates were validated by GPCR in combination with either the pharmacological Hh/Gli antagonist cyc or the agonist pur in Hh-responsive C3H10T1/2 cells. We also used small-hairpin RNAs against Gli proteins to evaluate targets and confirm specific Gli regulation their expression. Taking advantage of mutants that have been identified affecting different components of the Hh/Gli signaling system in the zebrafish model, we further analyzed specific novel candidates. Studying Hh function with pharmacological inhibition or activation complemented these genetic loss-of-function approaches. We provide evidence that in zebrafish embryos, Hh signaling regulates sfrp2, neo1, and c-myc expression in-vivo.
\end{abstract}

Conclusion: A recently described yeast-based screening allowed us to identify new Hh/Gli target genes, functionally important in different contexts of vertebrate embryonic development.

Keywords: Hh/Gli targets, zebrafish, purmorphamine, cyclopamine, neogenin 1, c-myc, sfrp2

\section{Background}

The Sonic hedgehog (Shh)/Gli pathway orchestrates several processes such as cell proliferation, differentiation, and stem cell maintenance [1]. Shh acts as a secreted protein ligand that binds to the 12-transmembrane receptor Ptc1 (Patched/Ptch/Ptch1). This binding releases Ptc1 repression of the 7 -transmembrane coreceptor Smoothened, which in turn favours intracellular accumulation of activator forms of the Gli transcription factors (Gli-A). In vertebrates, at least three Gli

\footnotetext{
* Correspondence: vpalma@uchile.cl

† Contributed equally

${ }^{1}$ Faculty of Sciences, Universidad de Chile, Santiago, Chile

Full list of author information is available at the end of the article
}

proteins (Gli1, Gli2 and Gli3) have been described. These zinc-finger transcription factors enter the nucleus and bind specific sequences (GACCACCCA) in the genome known as Gli Binding sites (GBS), promoting expression of target genes, including $p t c 1$ and gli1 themselves [2]. In the absence of ligand, Ptc1 represses Smoothened activity and the repressor forms of the transcription factors Gli (Gli-R) enter the nucleus and inhibit Hh target gene transcription. The relative ratio of Gli-A/Gli-R forms is considered to be crucial for interpreting the extracellular Hh gradient and for determining concentration-dependent cell fates [3,4]. Due the multiple developmental and growth processes where the $\mathrm{Hh} /$ Gli pathway has been implicated, it is likely that the
Ciomed Central

() 2012 Milla et al; licensee BioMed Central Ltd. This is an Open Access article distributed under the terms of the Creative Commons Attribution License (http://creativecommons.org/licenses/by/2.0), which permits unrestricted use, distribution, and reproduction in any medium, provided the original work is properly cited. 
cellular outcome involves a high number of target genes. To date, based on the identification of a Gli binding motif within their regulatory sequences, no more than a dozen target genes have been characterized to be activated or inhibited by Gli activity in vertebrates. Several in silico and experimental genomic analyses, such as ChIP-seq and ChIP-Chip, have been recently made to identify new gene direct targets, mainly identifying putative GBS near the transcriptional start site of genes [5-8]. However, it has been shown that different genomic strategies yield different hedgehog target lists.

In order to contribute to the identification of new GBS we applied a recently described yeast-based screen [9] using mouse and zebrafish genomic libraries. This versatile yeast strategy allowed us to rapidly and efficiently identify genomic targets of Gli-binding proteins. Bioinformatic analyses (MEME/MAST) were performed to determine the presence of enriched elements upstream or in the first intron of putative targets identified by our one-hybrid assay. Using this heterologous approach, we were able to identify several novel Glibinding sequences located close to genes previously not connected to the Shh/Gli pathway. Due to the high conservation of the $\mathrm{Hh} / \mathrm{Gli}$ pathway in vertebrates, some of the identified putative targets were further analyzed, both in-vitro using a mouse Hh reporter fibroblast cell line, and in-vivo in zebrafish embryos, confirming their predicted regulation through Hh loss and gain-of-function experiments. The high rate of identified target genes supports our unbiased approach and shows the potency of this method for finding novel target genes.

\section{Results}

\section{Yeast-based screens identify Gli protein-binding} sequences

In order to identify possible GBS, we used our recently described yeast-based assay that uses mouse and zebrafish libraries with random genomic fragments upstream of the yeast URA3 promoter [9]. The zebrafish library had an average genomic fragment size of $300 \mathrm{bp}$, and contains approximately $3 \times 10^{7}$ independent clones providing a 4- to 6-fold coverage of the genome, while the mouse library contains $1.7 \times 10^{7}$ independent clones with an average size of $700 \mathrm{bp}$, representing an average of 3 - to 4-fold coverage of the genome. MATa yeast containing the genome library fragment plasmids were mated separately to MAT $\alpha$ yeast containing an expression plasmid for either the full-length zebrafish Gli1 or the zinc-finger domain of the mouse Gli2 protein, following a standard two-hybrid mating protocol, screening afterwards a total of 611 clones [10]. Clones were then re-screened for a URA3 phenotype and the genomic fragment was PCR amplified using primers flanking the genomic library. Sequencing a total of 512 single product colony-PCR reactions, we successfully obtained 235 potential binding sequences for the mouse library, and 197 for the zebrafish library.

\section{MEME and MAST analysis predict multiple Shh/Gli target genes}

The consensus GBS, GACCACCCA, was first described by the Vogelstein group [11]. Several other genes that have been reported as targets of Gli1 in different biological processes, posses motifs with distinct degrees of similarity to the consensus sequences in their upstream regions. These motifs were able to bind a recombinant form of Gli1 in gel-shift assays [12]. From the 432 sequences obtained from the yeast assay, we manually curated them based on two criteria: first, we considered those sequences mapping within $20 \mathrm{~kb}$ of the transcriptional start of a gene or to the first intron, and second, we ruled out sequences that have been described as repetitive in multiple genome assemblies. We therefore did not consider the intergenic repetitive sequences, although they show tandem repeats that probably could bind the Gli transcription factors. Nevertheless, for the purpose of this study, they were discarded based on the fact that they do not show the Gli consensus binding sites or other known transcription factor binding sites. Next, we selected for further analysis and logo generation the mouse sequences since they fulfilled our request of mapping criteria. This yielded a list of 128 , mouse sequences, with final 66 non-redundant sequences (Additional File 1), mapped to specific genes based on shortest distance to the transcriptional start site. These sequences were used as input for a MEME search.

As shown in Figure 1A, we used the MEME [13,14] algorithm to identify overrepresented motifs in the group of 128 sequences (Additional File 1). A predicted site, a 14 bp motif (Figure 1A), contained the core consensus of GBS as described in [11]. The position-dependent scoring matrix for the $14 \mathrm{bp}$ motif was used as input to the MAST algorithm to search similar motifs in a $20 \mathrm{~kb}$ upstream region and in the first intron of 10 candidate genes, including $p t c 1$, candidate genes were selected based on gene ontology associations, to cover a wide array of cellular and developmental processes (Figure $1 \mathrm{C}$ ). Figure $1 \mathrm{~B}$ shows the representative logos of potential Gli-binding sites identified in the non-coding regions of the selected genes, for each gene the number of sequences that matched to the $14 \mathrm{bp}$ motif is indicated in the brackets right next to gene name.

We also checked the sequences for interspecies conservation, finding a high percentage for some of them in a murine/human comparison (like neo1 and cdh13), partial human/murine conservation for others (kif21a, rps6ka3), some with murine-only conservation (like 


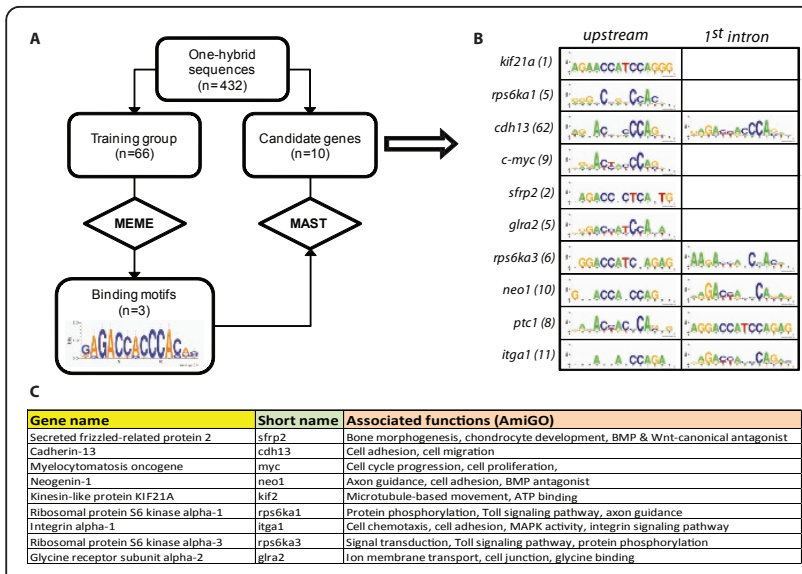

Figure 1 Motif search strategy. A) Diagram of the bioinformatic workflow for sequence analysis. A subgroup of sequences from the one-hybrid assay was used to search for motifs using MEME. Logos on the right represent the position-dependent matrix based in the alignment for GLI transcription factor candidate motifs. The matrix was also used as input to the MAST algorithm to identify potential Gli-binding sites in non-coding regions of candidate genes. B) Motifs identified with MEME/MAST strategy. Logos represent the alignment of the potential binding sites identified in each sequence in both upstream (left column) and intronic (right column) for nine candidate genes. Numbers of potential binding sites are indicated in brackets next to gene name. C) Gene ontology table for selected putative target genes found in (B)

itga1), and finally some with no conservation at all (sfrp2) (Additional File 2).

\section{Putative Gli-targets genes display altered mRNA levels after pharmacological blockage or activation of $\mathrm{Hh} / \mathrm{Gli}$ signaling pathway}

Assessing whether regulatory elements are occupied invivo requires experimental confirmation. In order to address whether any of the putative 9 target genes are affected by Hh pathway loss of function, we made use of the reporter cell line $\mathrm{C} 3 \mathrm{H} 10 \mathrm{~T} 1 / 2$ and assayed the candidate target genes for their response to $\mathrm{Hh}$ signaling by quantitative RT-PCR (qPCR). Cells were cultured in the presence of the $\mathrm{Hh} / \mathrm{Gli}$ pathway inhibitor cyclopamine (cyc) $(10 \mu \mathrm{M})$ and three time points were considered (6, 12 and 24 hours) to account for differences in the temporal transcriptional response of putative Gli-target genes and for differences in the stability of mRNAs produced under Gli control. Vehicle control (EtOH) was used as reference.

All the selected candidate genes showed reductions in their mRNA levels after 24-hour cyc treatments except glra (Figure 2). To corroborate $\mathrm{Hh}$ regulation we performed gain of function experiments using purmorphamine (pur), a proven Smoothened agonist [15] for a subset of candidates, namely neo1,c-myc and sfrp 2 based on their implication in developmental processes.
Cells were treated for 24 hours and 48 hours before RNA extraction and qPCR processing. ptc1, a well known Hh transcriptional readout was used as positive control. For both $c-m y c$ and neo1 significant changes could be observed after 24-hours of treatment, nevertheless the increases was less pronounced in comparison to ptc1. In order to proof that the effects of Hh signaling were direct we included experiments with cycloheximide (CHX), obtaining similar results (Additional File 3). Increase in transcript levels of the selected target genes became clearly evident after 48 hours of treatment. Sfrp2 did not show any changes in expression for either time point (Figure 3). sfrp 2 invariant mRNA levels possibly points to a context dependent case of regulation. Expression changes were calculated related to vehicle treatments (DMSO).

Based on the Gli-specific effect on transcription of novel target genes obtained by the screening and further in-vitro validation experiments for neo1, c-myc and sfrp2, we also verified Hh regulation of these genes by whole mount in situ hybridization (ISH) in zebrafish embryos. Besides allowing a relatively rapid verification of gene expression in different Hh-manipulated embryos, this approach provides spatial information and insights about the nature of regulation by Hh signaling. Because the Shh/Gli pathway plays a central role in neural development, particularly in the development of the central nervous system (CNS), we choose these known developmental genes not previously reported to be regulated by Shh signaling.

\section{Neogenin 1 is positively regulated by the Shh/Gli pathway in in the CNS}

Neogenin 1 is a multifunctional transmembrane receptor belonging to the immunoglobulin superfamily with known roles in angiogenesis, progenitor proliferation and axon guidance [16]. At 48 hours post fertilization (hpf) neo1 expression was strongly detected in the wildtype CNS, where it was associated with proliferative zones (optic tectum, eye and diencephalon, Figure 4AE). Of note, neo1 was also detected in the notochord (Figure 4D, bracket). ISH analysis shows no staining in the notochord and the retinal ganglion cell layer of the eye in $d t r$ (gli1) and smu (smo) mutants (Figure 4F-I, bracket and arrowhead).

In addition to genetic loss-of-function, we complemented our analysis by treating embryos with the Shh/ Gli activator pur. Of note, in particular notochord expression is increased when compared to mock DMSO treated embryos (Figure 4J, K, bracket).

\section{c-myc is regulated by the Shh/Gli pathway in the CNS and liver}

We next asked whether $c-m y c$ ( $m y c a)$, a multifunctional gene directly related to cell cycle progression (G1/S 


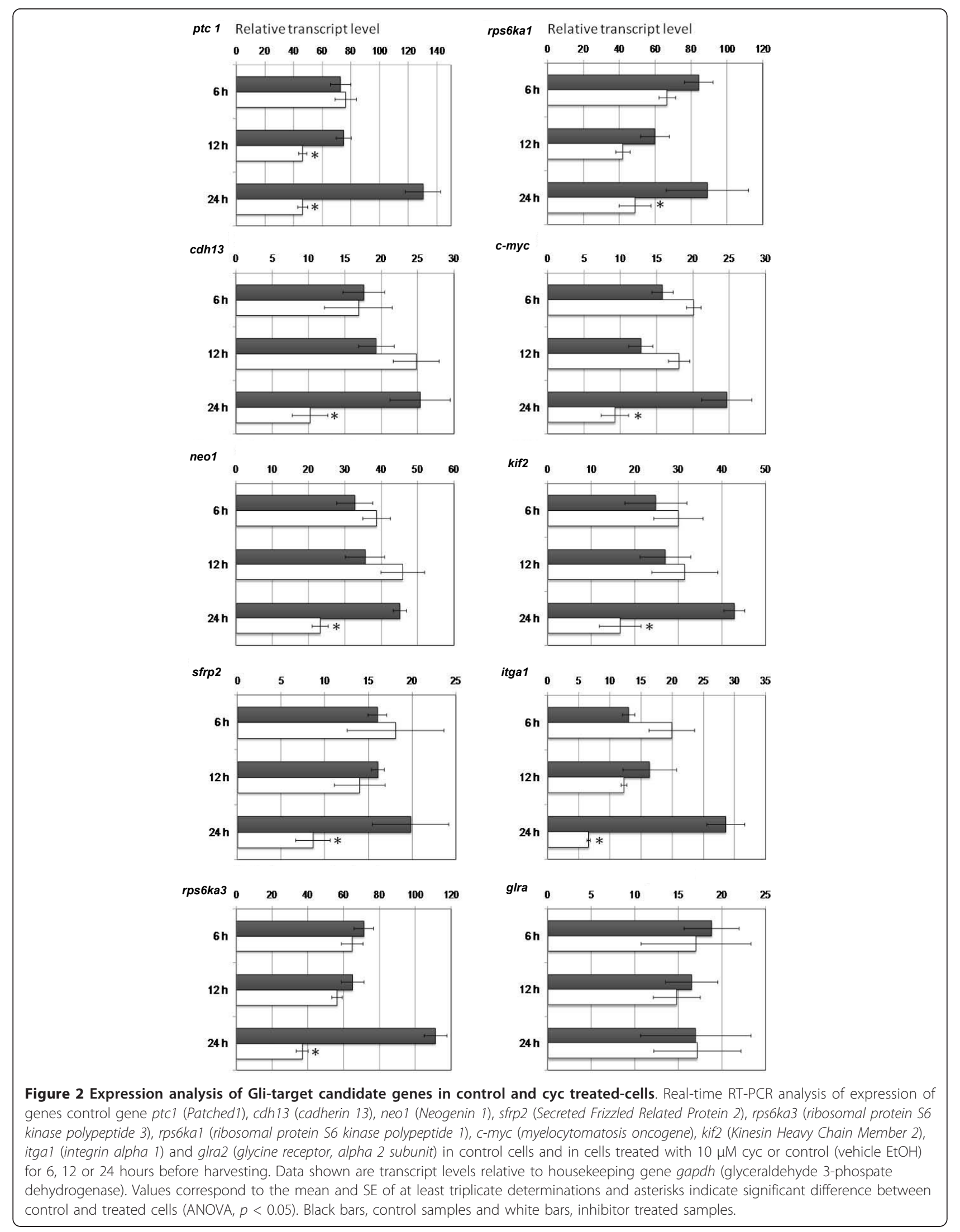




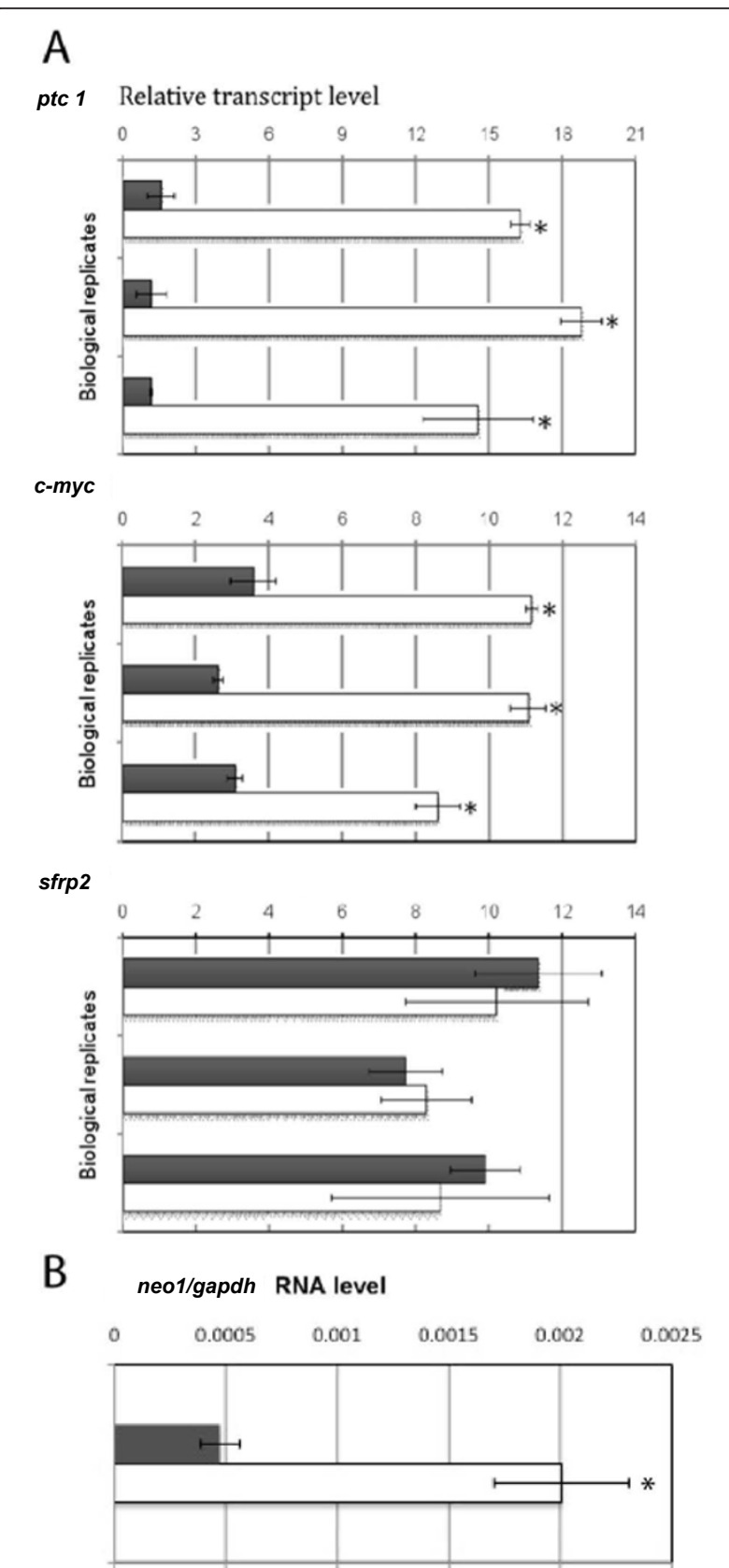

Figure 3 Pharmacological gain of function of the Shh/Gli pathway activates transcription of novel target genes. A)

C $3 \mathrm{H} 10 \mathrm{~T} 1 / 2$ cells were cultured for $48 \mathrm{~h}$ in $10 \mu \mathrm{M}$ pur or its control (vehicle DMSO) and processed for qPCR. c-myc shows significant differences in mRNA levels, whereas sfrp2 does not. ptc 1 is shown as a positive control. B) neol mRNA levels are increased after the same $48 \mathrm{~h}$ treatment of $\mathrm{C} 3 \mathrm{H} 10 \mathrm{~T} 1 / 2$ cells, graph showed separately due to use of TaqMan probes

phases) and implicated in a variety of cancers, is regulated by Shh [17]. At $48 \mathrm{hpf}$ wild-type $c-m y c$ expression is found in the posterior margin of the optic tectum, recently identified as an important proliferative zone in

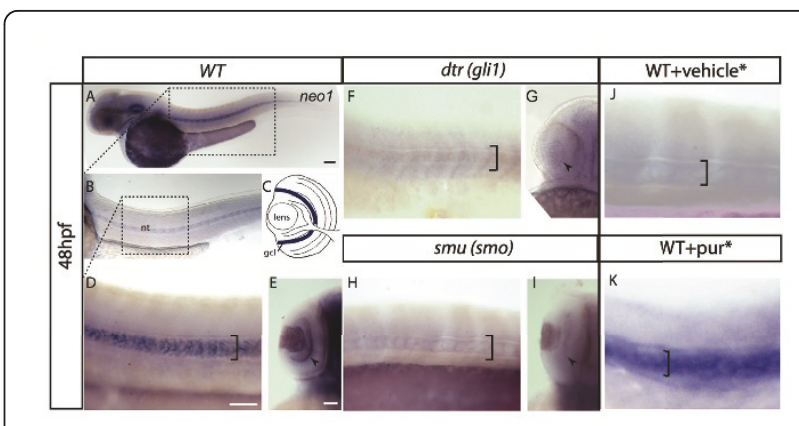

Figure 4 neo 1 expression is reduced in Shh/Gli pathway mutants and increased on Shh/Gli pharmacological activation. neo 1 is expressed broadly in the developing CNS (A). Expression in $48 \mathrm{hpf}$ embryo can be detected in the otic vesicle, in the notochord (B, close-up $D$, black bracket) and in the eye (E, black arrowhead), probably in the retinal ganglion cell layer ( $\mathrm{gll}$ ). (C) Cartoon of a $48 \mathrm{hpf}$ zebrafish eye. Blue line shows neo 1 expression in the gcl. At $48 \mathrm{hpf}$ neol expression is diminished in gli1 (dtr) and smu (smo) mutants, specifically in the notochord ( $F, H$, bracket) and the gcl in the optic placode (G, l; black arrowhead) in comparison to WT (D, E). (J, K) neo1 mRNA is increased in notochord. Note that $(J)$ and $(K)$ controls were developed at different times in comparison to (D) in order to match to their corresponding pur treatment (K). Anterior is towards left, lateral views are showed except in $(E, G, I, K)$ with dorsal views anterior up. Scale bars; $50 \mu \mathrm{m}$ except in A (100 $\mu \mathrm{m})$. WT; wild type. gcl; retinal-ganglion cell layer. DMSO is the vehicle for pur.

the dorsal brain of teleosts (Figure 5A, arrow) $[18,19]$. The mRNA can also be detected in the eye and liver (Figure 5A asterisk). In the $s m u$ mutant, expression in CNS and liver was completely diminished (Figure 5B). $d t r$ mutants did not show any $c-m y c$ staining; expression is regionally lost in the dorsal midbrain, eye and liver (Figure 5C, arrow indicates eye) whereas the yot (gli2) mutant has a reduced expression in the eye while $c-m y c$ was still detectable in the tectum and liver (Figure 5D). Thus, ISH analysis corroborated the changes in $c-m y c$ expression levels that were previously determined by qPCR assays.

\section{sfrp2 gene is regulated by the Shh pathway in slow muscle and pharyngeal arches}

The sfrp 2 gene encodes a protein that binds Wnt ligands through a cystein-rich domain (CRD). It has been implicated in both antagonism of Wnt signaling [20] and beta-catenin stabilization [21]. ISH analysis verified that $s f r p 2$ transcripts can be detected in adaxial cells, pectoral fins and branchial arches by the $48 \mathrm{hpf}$ stage, as previously reported [22]. Adaxial cell expression is absent in yot mutants, and expression is also regionally lost in the CNS (Figure 6B, B"'). Pectoral fins do not display any changes in sfrp 2 expression. This is consistent with the reported alterations in myogenesis in yot mutants, where slow muscle genesis is affected [23]. 


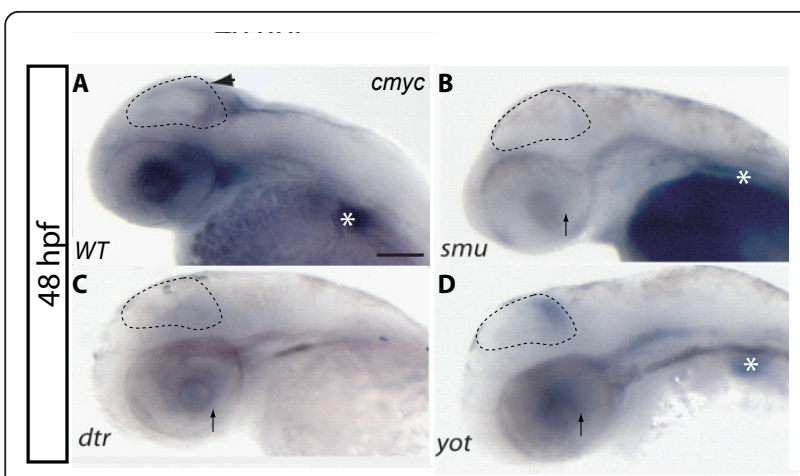

Figure 5 C-myc expression is lost in the CNS in Shh/Gli pathway mutants. A) c-myc is normally expressed in the optic tectum (black arrowhead) and the developing eye. Strong expression is also detected in the liver (white asterisk). B) smu mutant embryos show no expression of c-myc in the CNS. C) $d t r$ embryos have no c-myc in the eye (black arrow), tectum and liver D) yot embryos still show labelling for c-myc, although reduced; in both the eye and optic tectum labelling is preserved. Liver also retains some c-myc labelling. All lateral views are dorsal up, anterior left.

While it is known that a gradient of Wnt signaling opposes the Shh signaling gradient in the neural tube, regulation of $s f r p 2$ in the brain by Shh has not previously been demonstrated.

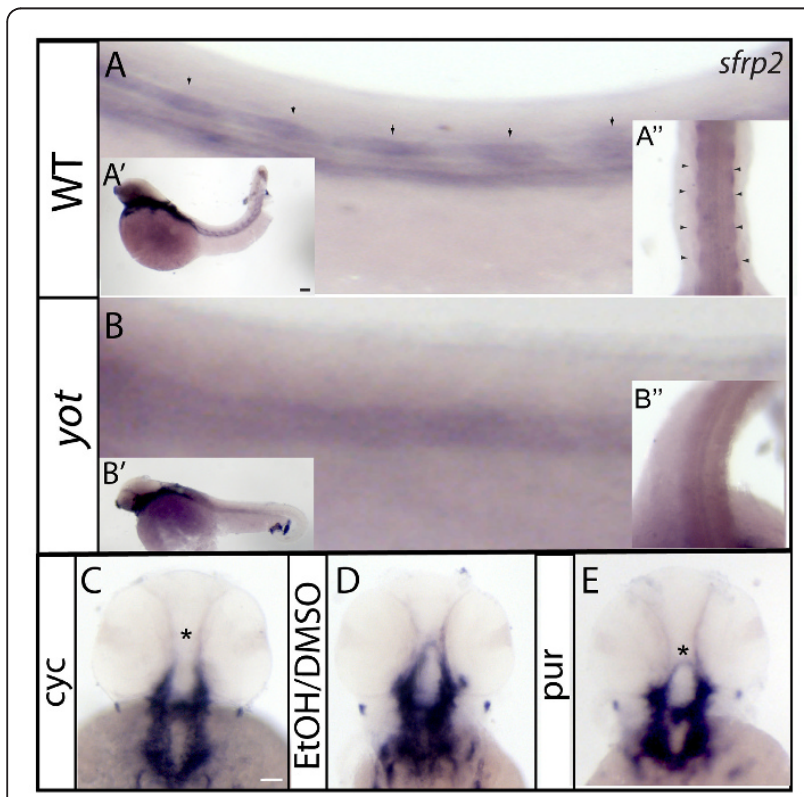

Figure 6 sfrp2 RNA levels are disturbed in Hh pathway mutants and after pharmacological treatments. (A, $\left.A^{\prime \prime}\right)$ In WT embryos, sfrp2 expression is evident in adaxial cells. This expression is significantly down-regulated in yot embryos $\left(B, B^{\prime \prime}\right)$.

Pharmacological treatments with pur and cyc show altered expression in pharyngeal arches (asterisk in E, compare C, D and E for different levels of RNA). Anterior is towards left, lateral views are shown. Full lateral views of embryos are shown in $A^{\prime}$ and $B^{\prime}$.
To further examine whether sfrp 2 is regulated by Shh we used pharmacological gain and loss of function approaches. Sfrp2 expression is increased in pur treated embryos in comparison to its vehicle treatment (Figure $6 \mathrm{E})$. Although cyc inhibitor does not ablate completely expression in the CNS, expression in the developing pharyngeal arch is abolished. (Figure 6C, see asterisk). This might be due to the fact that treatments were started at $8 \mathrm{hpf}$, due to an extremely high mortality rate in treatment initiated prior to that time. The failure to detect sfrp 2 regulation in the first reporter cell assay demonstrates the necessity to test regulation in the proper, in-vivo context.

\section{Discussion}

The Hedgehog pathway has been extensively studied. Many studies take advantage of high throughput approaches at different levels, for example by identifying the post-translational modifiers mediating Hh signaling [24], by mapping GBS and Gli responding genes in silico [25] or in different cellular models [26]. In general, ChIP assays utilize specific PCR primers flanking a suspected binding site to determine whether the site is occupied. Still, recent studies suggest that these regions, at least for mammalian genomes, are more complex than previously imagined and therefore ChIP-on chip may miss some regulatory regions. ChIP-Seq is another approach, but like ChIP-on chip, it requires high quality antibodies to the transcription factor of interest. The latter has been a big handicap particularly in the HH/Gli field; the development of ChIP grade of Gli antibodies is still a matter of intense research. To improve the number of target genes associated with the Shh/Gli pathway, we performed a yeast-based genomic screening assay combined with in silico analysis in order to characterize novel Gli interacting sequences. Yeast-based method has been successfully applied to identify transcription factor target genes in [9].

\section{Identifying new Hh targets using a yeast-based method}

Our strategy shows that sequences obtained by the yeast based method possess potential binding sites for Gli transcription factor whose consensus matches the $9 \mathrm{bp}$ core previously described. This method has the advantage that it allows transcription binding site identification for GBS that potentially cannot be found using other strategies. Of note, we found fragments that contain putative GBS sequences slightly different from what has been previously reported as consensus sequences (Figure 1B). Variations of these GBS lead us to examine whether a correlation existed between these changes and DNA binding domains for Gli family members. Zinc fingers 4 and 5, responsible for binding to the consensus sequence [27], are highly conserved specifically 
in the residues involved in contact with bases or phosphates. Lack of correlation between changes in individual consensus GBS sequences and DNA binding domains lead us to propose that these motifs might correspond to non-consensus binding sites for Gli1 with different affinities for the transcription factor. This hypothesis has been partially addressed in other works. In [25] for example, mutations of consensus GBS has been useful to predict potential targets for Gli family in mammalian enhancers, based in the effect of these mutations on activation of a luciferase reporter. These data indicate that variations from consensus GBS can affect gene expression levels, as also described in [6]. In [28], an essential binding site from ptc1 promoter, with a single substitution from the consensus GBS sequence, was mutated to investigate the effect of change bases in the expression of a reporter gene, resulting in different non-consensus and low affinity Gli binding sites that could lead to transcriptional activation.

Several models have been proposed for Hh activation of target genes. Gli proteins can bind to regulatory regions and directly either activate or repress transcription. In Drosophila [29] a differential affinity model seems to represent $\mathrm{Hh} / \mathrm{Ci}$ signaling in the wing imaginal disc. It remains to be seen whether Hh signaling in vertebrates follows a similar mechanism. We cannot rule out a role of Gli repressors (Gli3 in particular) in the physiological regulation of the new Gli target genes identified in this paper. The fact that we see increased expression of $p t c 1$, however, leads us to associate this regulation preferentially to Gli activator function. It remains to be defined whether Gli repressor assays yield the same targets as Gli activators.

A very interesting result of our work is the fact that we were able to mate yeast zebra fish or mouse Gli constructs to either homologous or heterologous libraries. Indeed, this reveals potential conservation of the interaction between the Gli transcription factors and their putative GBS among vertebrates. Multi-species conservation of the identified sequences was also addressed. The majority of the assayed genes showed low percentage of mice-rat-human similarity or murine only conservation. Some genes like neo1 showed a high percentage of conservation in mice-rat-human comparisons, while others like sfrp 2 did not show any conservation, probably due to the partial presence of repeating elements. Some of these sequences, though, also show predicted repeating elements, which might account for the absence of conservation observed in the multiple alignments.

\section{Target gene expression}

Further experiments using the Hh-responsive cell line C3H10T1/2 confirmed the results obtained from our screening, showing positive correlations of expression level loss to pharmacological loss of function of the $\mathrm{Hh} /$ Gli pathway. Interestingly, though, none of our chosen genes show an "early response" to cyc treatments, with reduced mRNA levels coming after 12 hours of treatment like $p t c 1$. Instead they displayed a slower response to pathway inhibition, where expression is significantly reduced only after 24 hours of treatment. Similarly, 24 hours pur treatment showed a rather modest increase in target genes, such as neo1 and $c-m y c$, in comparison to the well known described ptc1 up-regulation. This may suggest different levels of regulation, possibly due to the different genomic and regulatory contexts of the given promoters causing a different response dynamic than ptc1. Indeed, it has been reported that the expression of some $\mathrm{Hh}$ target genes depends on pathway activation (e. g. that of gli1), whereas other targets must be expressed prior to the induction of Gli activators, which then have their expression increased via positive feedback (e.g. ptc1). Thus, it will be interesting to discover possible similarities and differences in the transcription of different types of Hh target genes.

Our results clearly indicate the necessity for functionally testing individual genes and associated networks resulting from bioinformatics analysis. That some of the selected targets do not show increased mRNA levels when cells are treated with the pur agonist (like sfrp2) is likely due to additional signals regulating gene expression. Gain-of-function provides extra information on the mechanisms of Gli-mediated transcription of these target genes when compared to loss-of-function treatments. The fact that seven out of eight of the chosen genes show reduced mRNA after cyc treatment highlights the degree of accuracy that our screen assay provides for identifying target genes and corroborates our selection criteria.

A major finding of this study is the identification of several novel Shh target genes that we show to be important for embryonic development in-vivo. The use of zebrafish embryos as an in-vivo model to validate target genes has been previously reported [7], and our ISH provided a spatial-temporal resolution to changes observed at the transcript level in cell culture. Here we tested three candidate genes, selected based on their involvement in different processes during development. sfrp 2 is involved in myoblast differentiation [30]. c-myc is involved in the cell cycle and critically regulates cell proliferation [31]. neo1 has been related to axon migration and more recently to neural progenitor proliferation in the CNS [32]. The Shh pathway has been classically implicated in both myogenesis and neurogenesis. Indeed, Shh loss of function in early development generates severe alterations in slow muscle development [33]. Patterning of the dorsal/ventral axis, specification of 
neuronal and oligodendroglial cell types, and proliferation of dorsal brain structures like the cerebellum, optic tectum and the neocortex also rely on Shh signaling $[6,18]$. In addition to the key functions of Shh in CNS development, is also implicated in oncogenesis. Shh signaling is deregulated in medulloblastomas (MB), embryonic tumors composed of primitive-appearing cells that arise in the cerebellum. Interestingly, a recent survey of the methylation status of tumor suppressors or oncogenes in human MBs has revealed among several epigenetic silencers sfrp family members [34].

All chosen candidate genes evaluated in the zebrafish embryo showed altered expression consistent with the in-vitro data obtained from $\mathrm{C} 3 \mathrm{H} 10 \mathrm{~T} 1 / 2$. Their response in-vivo in the zebrafish embryo was a very context dependent response, with expression showing variation in some structures while remaining unaltered in others. Not all expression was ablated in smu mutants, however, so this might pertain to additional signals that could be regulating expression in a context-dependent manner in the zebrafish embryo. All three Gli family members have been shown to recognize the same GBS and share redundant functions. However, our data clearly show functional differences between Gli1 and Gli2 members, as evidenced by the zebrafish mutant analysis. Thus it is of great interest to further investigate the specific functions of each Gli transcription factor, sorting out their distinct and overlapping roles upon targets.

\section{Conclusions}

Our study uncovered target genes previously linked to Shh signaling and, most importantly, previously unknown genes likely to play important roles during embryonic development. Several new candidates, including positive controls, whose modulation by Shh/Gli has been reported, were analyzed in the context of zebrafish development. These findings extend our knowledge of the Shh regulatory pathway function in development. Attaining a thorough understanding of $\mathrm{Hh}$ signaling is of vital importance for developing a mechanistic understanding of congenital abnormalities and diseases.

\section{Methods}

Yeast growth, complementation and colony sequencing Yeast protocols (growth and mating) were performed as described in [9]. Plasmids used for yeast libraries are the same as in [9]. Sequencing was performed as described in [9].

\section{MEME \& MAST analysis}

Intronic and upstream regions were obtained either through the ENSEMBL database or the UCSC genome browser database. Motif searches were performed using standalone MEME suite software available at http:// meme.sdsc.edu/meme/cgi-bin/meme.cgi. MEME was run with the following parameters: -dna -mod tcm -nmotifs 3 -minw 6 -maxw 14 -evt 1 e100 -revcomp -time 7200 -maxsize 60000 -nostatus -maxiter 20. MAST was running using -remcorr and -norc parameters.

\section{Clustal alignment}

Sequence from Gli1, Gli2 and Gli3 proteins were obtained from the ENSEMBL database. Alignment was performed with the Bioedit editor [9], with default options for Clustal. For Gene Ontology analysis, we used DAVID at http://david.abcc.ncifcrf.gov/home.jsp and AmiGO at http://amigo.geneontology.org/.

\section{C3H10T1/2 cell culture and treatment}

The cell line was obtained from ATCC. Cells were grown in Dulbecco's Modified Eagle Media (DMEM) supplemented with 10\% Fetal Bovine Serum (FBS). Cells were passaged at $80 \%$ confluence and passages 2 through 9 were used. Gain and loss of function experiments were performed according to [35] Briefly, considering that these cells are highly contact inhibited we performed experiments once the cell projections are touching, but before full confluence (aprox. 60\% confluence). Cells were not serum deprived before treatments; cyc was added in presence of $10 \%$ FBS, whereas pur was given in $0.5 \%$ FBS. Cells were treated for $24 \mathrm{hrs}$ in presence of $0.5 \%$ FBS with either $1.25 \mu \mathrm{g} / \mathrm{mL}$ of the protein synthesis inhibitor $\mathrm{CHX}$ alone or in combination with the Hh agonist.

\section{Quantitative real-time PCR (qPCR)}

RNA was extracted using TRI Reagent (Ambion) according to the manufacturer's recommendations. RNA integrity and purity were electrophoretically verified by ethidium bromide staining and by OD260/OD280 nm absorption ratio. RNAs were then treated with Turbo DNA-free kit (Ambion) and reverse transcribed with Oligo-dT and Superscript II (Invitrogen). Transcripts of Bacillus subtilis dap gene were added to the total RNA of each sample $(1: 2,000)$ as spike-in controls to monitor the efficiency of reverse transcription. To generate the spike-in control RNA, the plasmid containing B. subtilis dap gene was purified from strain ATCC 87486. Linear template DNA was generated by digesting the plasmid with restriction enzyme NotI and RNA was produced by in-vitro transcription using T3 RNA polymerase. The specific forward and reverse oligonucleotide primers for target genes and for reference gene (Additional file 4) were designed using Primer Premier 5.0 software (Premier Biosoft International), based on GeneBank database sequences. Quantitative RT-PCR (qPCR) reactions were performed in a LightCycler system (Roche) using SYBR 
Green to monitor cDNA amplification. The amplification reaction contained: $1 \mu \mathrm{L}$ of LightCyclerTM DNA Master SYBR ${ }^{\circledR}$ Green I, $25 \mathrm{mM} \mathrm{MgCl2,} 10 \mu \mathrm{M}$ of forward and reverse primers and $100 \mathrm{ng}$ of cDNA in a total volume of $10 \mu \mathrm{L}$. The following standard thermal profile was used: denaturation at $95^{\circ} \mathrm{C}$ for $10 \mathrm{~min}$, followed by 35 three-step cycles of template denaturation at $95^{\circ} \mathrm{C}$ with a $5 \mathrm{~s}$ hold, primer annealing at $58-64^{\circ} \mathrm{C}$ for $10 \mathrm{~s}$ and extension at $72^{\circ} \mathrm{C}$ for $10 \mathrm{~s}$. Data were analyzed using the LightCycler Software (v3, Roche). The reaction efficiency was determined for each PCR reaction with LinRegPCR v7.5 [36]. Three technical replicates were done for each combination of CDNA and primer pair, and the quality of the PCR reactions was checked through analysis of the dissociation and amplification curves. The products were resolved by $2 \%$ agarose gel electrophoresis to confirm the DNA fragments of expected size. Transcript levels of target genes within a cDNA were normalized to the respective transcript level of gapdh as described in [37]. To test whether gadph behaves as a housekeeping gene in the analyzed samples, transcript levels of gadph and dap were measured by qPCR in each cDNA sample and the ratios of control transcript to the endogenous transcript gadph were calculated. The results indicated that the abundance of gadph mRNA remains stable between samples (data not shown). qPCR reactions were performed on material from at least two independent biological experiments. To assess differences among treatments, we applied a one-way ANOVA and a posteriori LSD Fisher test. A pvalue $<0.05$ was considered statistically significant. Data from the CHX experiment were processed as described above, except that results were expressed as a ratio of relative transcript levels between control and treated cells.

\section{Zebrafish embryos}

Wild-type and mutant zebrafish embryos were maintained at $28^{\circ} \mathrm{C}$ as described in the Zebrafish Book [38], and staged accordingly [39]. Mutant lines used were smooth-muscle omitted (smuhi1640; [40,41], a loss-offunction smoothened allele, detour (dtrts269; [41]), a loss-of-function gli1 allele and you-too (yot; [42]), a negative dominant allele for gli2. Mutants were maintained as heterozygotes and heterozygous adults were crossed to produce homozygous mutant offspring. Homozygous mutant individuals were identified by morphological criteria ( $d t r$ and yot). Developmental time points are expressed as hours post-fertilization (hpf).

All animal procedures were in accordance with the Chilean legislation and were approved by Institutional Animal Care and Use Committees.

\section{RNA in situ hybridization}

In situ hybridization was performed essentially as previously described [43]. Partial neogenin1 (neo1) cDNA was isolated by PCR and a digoxigenin-labelled antisense neo1 probe was synthesized from the cDNA clone. The clone was linearized with ApaI and transcribed with T7 polymerase (Roche, Hertfordshire, UK). c-myc cDNA clone was a kind donation from Dr. Nora Calcaterra. Plasmid was digested with EcoRI then antisense probe generated using T7 polymerase. sfrp2 clone, a donation from Dr. Corinne Houart, linearized with EcoRI and anti-sense probe transcribed using $\mathrm{T} 7$ polymerase.

\section{Pharmacological treatments}

Zebrafish embryos were treated with $10 \mu \mathrm{M}$ cyc (Infinity Pharmaceuticals, Inc., Cambridge, MA) by adding $1 \mu \mathrm{l}$ of a $10 \mathrm{mM}$ stock solution (in $95 \% \mathrm{EtOH}$ ) to $1 \mathrm{ml}$ of $\mathrm{E} 3$ medium at defined time points. Control embryos were treated simultaneously with an equal volume $(1 \mu \mathrm{l})$ of 95\% EtOH (cyc carrier). For pur (Calbiochem, CA) treatments embryos were grown in E3 medium with 10 $\mu \mathrm{M}$ of the $\mathrm{Hh}$ agonist at indicated time points and control embryos were treated with 1\% DMSO in E3 medium. Treatments were carried out in 6 -well plates in 2 $\mathrm{mL}$ E3 medium (30 embryos/well) at $28.5^{\circ} \mathrm{C}$. Embryos were fixed with $4 \%$ paraformaldehyde, dehydrated in $\mathrm{MeOH}$ and processed for in situ hybridization and/or antibody labelling. Effectiveness of cyc was verified by decreased ptc1 expression of treated embryos while pur showed to be specific to Hh signaling, as indicated by increased ptc1 expression (shown in Additional File 5).

\section{Imaging}

Photographs were either taken with a Leica DFC 300 FX camera using a Leica MZ12 dissecting microscope. Images were processed with Photoshop 7.0 for Macintosh and Image $\mathrm{J}(\mathrm{NIH})$.

\section{Additional material}

Additional file 1: Table listing all binding sites found in Shh/Gli yeast assay through our screening strategy. Total mouse GBS positive sequences mapped to specific genes based on shortest distance to the transcriptional start sites.

Additional file 2: GBS interspecies conservation. in silico analysis of GBS found in the promoter of selected vertebrate genes.

Additional file 3: Direct regulation of novel Shh/Gli target genes in the CH310T1/2 murine cell line. CHX 24 hours treatment confirm direct regulation for canonical Shh regulated genes.

Additional file 4: Table listing all primers used in this study. Primers designed for control and novel Shh/Gli target gene quantitative PCR assays.

Additional file 5: Analysis of in-vivo drug response based on ptc1 expression. Control of zebrafish long-term pharmacological Hedgehog 
gain and loss of function treatment. ptcl readout-gene expression was verified by in situ hybridization.

\section{Abbreviations}

Hh: Hedgehog; Shh: Sonic Hedgehog; TC: Optic tectum; CNS: Central Nervous System; Ptc1: Patched1; Smo: Smoothened; WT: Wild type; hpf: hours post fertilization; smu: slow-muscle-omitted; dtr: detour; yot: you too; cyc: cyclopamine; qPCR: quantitative Real Time PCR.

\section{Acknowledgements}

We would like to thank Catalina Lafourcade for technical assistance, Dr. Miguel Allende for kindly providing access to zebrafish embryos and the zebrafish community for reagents and Hh mutant lines. We are particularly grateful to the PEW foundation for their continuous support. This work was supported by FONDAP 15090007 (VP, VC). Fondecyt grant 1070248, 1110237 (VP), Fondecyt Postdoctoral 3100045 (LAM), Fondecyt 1090211 (VC) and Intramural Research Program of the National Human Genome Research Institute, National Institutes of Health (SB).

\section{Author details}

${ }^{1}$ Faculty of Sciences, Universidad de Chile, Santiago, Chile. 'Laboratorio de Bioinformática y Expresión Génica, INTA, Universidad de Chile, Santiago, Chile. ${ }^{3}$ FONDAP Center for Genome Regulation, Facultad de Ciencias, Universidad de Chile. ${ }^{4}$ National Human Genome Research Institute, National Institutes of Health, Bethesda, Maryland, USA

\section{Authors' contributions}

LAM, CC and VP designed the experiments and drafted the manuscript. LAM performed the yeast based screening. CHQ, VC and LAM performed the expression experiments in cell culture, data analyses, and revised the manuscript. CC, MO and LAM performed the zebrafish expression experiments. SB designed the yeast-based screenings and revised the manuscript All authors have read and approved the final manuscript

Received: 23 May 2011 Accepted: 3 January 2012

Published: 3 January 2012

\section{References}

1. Barakat MT, Humke EW, Scott MP: Learning from Jekyll to control Hyde: Hedgehog signaling in development and cancer. Trends in Molecular Medicine 2010, 16(8):337-48.

2. Sasaki H, Hui C, Nakafuku M, Kondoh $H$ : A binding site for Gli proteins is essential for HNF-3beta floor plate enhancer activity in transgenics and can respond to Shh in vitro. Development 1997, 124:1313-22.

3. Bai $C B$, Stephen D, Joyner AL: All mouse ventral spinal cord patterning by hedgehog is Gli dependent and involves an activator function of Gli3. Developmental Cell 2004, 6:103-15.

4. Persson $M$, Stamataki $D$, te Welscher $P$, et al: Dorsal-ventral patterning of the spinal cord requires Gli3 transcriptional repressor activity. Genes \& Development 2002, 16:2865-78.

5. Vokes SA, Ji H, McCuine S, et al: Genomic characterization of Gli-activator targets in sonic hedgehog-mediated neural patterning. Development 2007, 134:1977-89.

6. Lee EY, Ji H, Ouyang Z, et al: Hedgehog pathway-regulated gene networks in cerebellum development and tumorigenesis. Proceedings of the National Academy of Sciences of the United States of America 2010, 107:9736-41.

7. Bergeron $S A$, Milla LA, Villegas $R$, et al: Expression profiling identifies novel $\mathrm{Hh} / \mathrm{Gli}$-regulated genes in developing zebrafish embryos. Genomics 2008, 91:165-77.

8. Vokes SA, Ji H, Wong WH, McMahon AP: A genome-scale analysis of the cis-regulatory circuitry underlying sonic hedgehog-mediated patterning of the mammalian limb. Genes \& Development 2008, 22:2651-2663.

9. Zeng J, Yan J, Wang T, et al: Genome wide screens in yeast to identify potential binding sites and target genes of DNA-binding proteins. Nucleic Acids Research 2008, 36:e8.
10. Soellick TR, Uhrig JF: Development of an optimized interaction-mating protocol for large-scale yeast two-hybrid analyses. Genome Biology 2001 2:RESEARCH0052.

11. Kinzler KW, Vogelstein B: The GLI gene encodes a nuclear protein which binds specific sequences in the human genome. Molecular and Cellular Biology 1990, 10:634-42.

12. Yoon JW, Kita Y, Frank DJ, et al: Gene expression profiling leads to identification of GLI1-binding elements in target genes and a role for multiple downstream pathways in GLI1-induced cell transformation. The Journal of Biological Chemistry 2002, 277:5548-55.

13. Bailey $\mathrm{TL}$, Gribskov M: Combining evidence using p-values: application to sequence homology searches. Bioinformatics 1998, 14:48-54.

14. Bailey $T L$, Elkan C: Fitting a mixture model by expectation maximization to discover motifs in biopolymers. Proceedings/... International Conference on Intelligent Systems for Molecular Biology; ISMB. International Conference on Intelligent Systems for Molecular Biology 1994, 2:28-36.

15. Sinha S, Chen JK: Purmorphamine activates the Hedgehog pathway by targeting Smoothened. Nature Chemical Biology 2006, 2:29-30.

16. Wilson NH, Key B: Neogenin: one receptor, many functions. The International Journal of Biochemistry \& Cell Biology 2007, 39:874-8.

17. Vita M, Henriksson M: The Myc oncoprotein as a therapeutic target for human cancer. Seminars in Cancer Biology 2006, 16:318-30.

18. Chaplin N, Tendeng C, Wingate RJT: Absence of an external germinal layer in zebrafish and shark reveals a distinct, anamniote ground plan of cerebellum development. The Journal of Neuroscience: the official journal of the Society for Neuroscience 2010, 30:3048-57

19. Feijóo CG, Oñate MG, Milla LA, Palma VA: Sonic hedgehog (Shh)-Gli signaling controls neural progenitor cell division in the developing tectum in zebrafish. The European Journal of Neuroscience 2011, 33:589-98.

20. Lee CS, Buttitta LA, May NR, Kispert A, Fan CM: SHH-N upregulates Sfrp2 to mediate its competitive interaction with WNT1 and WNT4 in the somitic mesoderm. Development 2000, 127:109-18.

21. Kress E, Rezza A, Nadjar J, Samarut J, Plateroti M: The frizzled-related sFRP2 gene is a target of thyroid hormone receptor alpha1 and activates betacatenin signaling in mouse intestine. The Journal of Biological Chemistry 2009, 284:1234-41.

22. Tendeng C, Houart C: Cloning and embryonic expression of five distinct sfrp genes in the zebrafish Danio rerio. Gene Expression Patterns: GEP 2006, 6:761-71

23. Roy S, Wolff C, Ingham PW: The u-boot mutation identifies a Hedgehogregulated myogenic switch for fiber-type diversification in the zebrafish embryo. Genes \& Development 2001, 15:1563-76

24. Varjosalo $M, B j o ̈ r k l u n d ~ M, C h e n g ~ F$, et al: Application of active and kinasedeficient kinome collection for identification of kinases regulating hedgehog signaling. Cell 2008, 133:537-48.

25. Hallikas O, Palin K, Sinjushina N, et al: Genome-wide prediction of mammalian enhancers based on analysis of transcription-factor binding affinity. Cell 2006, 124:47-59.

26. Varjosalo M, Li S-P, Taipale J: Divergence of hedgehog signal transduction mechanism between Drosophila and mammals. Developmental Cell 2006, 10:177-86.

27. Pavletich NP, Pabo CO: Crystal structure of a five-finger GLI-DNA complex: new perspectives on zinc fingers. Science 1993, 261:1701-7.

28. Winklmayr M, Schmid C, Laner-Plamberger S, et al: Non-consensus GLI binding sites in Hedgehog target gene regulation. BMC Molecular Biology 2010, 11:2

29. Parker DS, White MA, Ramos Al, Cohen BA, Barolo S: The cis-regulatory logic of Hedgehog gradient responses: key roles for gli binding affinity, competition, and cooperativity. Sci Signal 2011, 4:ra38.

30. Descamps S, Arzouk H, Bacou F, et al: Inhibition of myoblast differentiation by Sfrp1 and Sfrp2. Cell and Tissue Research 2008 332:299-306.

31. Pelengaris $\mathrm{S}$, Khan M, Evan G: c-MYC: more than just a matter of life and death. Nature reviews Cancer 2002, 2:764-76.

32. Vries M, De Cooper HM: Emerging roles for neogenin and its ligands in CNS development. Journal of Neurochemistry 2008, 106:1483-92.

33. Du SJ, Dienhart M: Gli2 mediation of hedgehog signals in slow muscle induction in zebrafish. Differentiation; research in biological diversity 2001, 67:84-91. 
34. Kongkham PN, Northcott PA, Croul SE, et al: The SFRP family of WNT inhibitors function as novel tumor suppressor genes epigenetically silenced in medulloblastoma. Oncogene 2010, 29:3017-24.

35. Ingram WJ, Wicking CA, Grimmond SM, Forrest AR, Wainwright BJ: Novel genes regulated by Sonic Hedgehog in pluripotent mesenchymal cells. Oncogene 2002, 21:8196-205.

36. Ramakers C, Ruijter JM, Deprez RHL, Moorman AFM: Assumption-free analysis of quantitative real-time polymerase chain reaction (PCR) data. Neuroscience Letters 2003, 339:62-6.

37. Talke IN, Hanikenne M, Krämer U: Zinc-dependent global transcriptional control, transcriptional deregulation, and higher gene copy number for genes in metal homeostasis of the hyperaccumulator Arabidopsis halleri. Plant Physiology 2006, 142:148-67.

38. Westerfield M: The zebrafish book. A guide for the laboratory use of zebrafish (Danio rerio). 4 edition. Eugene: Univ. of Oregon Press; 1993.

39. Kimmel CB, Ballard WW, Kimmel SR, Ullmann B, Schilling TF: Stages of embryonic development of the zebrafish. Developmental Dynamics: an official publication of the American Association of Anatomists 1995, 203:253-310

40. Stickney HL, Barresi MJ, Devoto SH: Somite development in zebrafish. Developmental Dynamics: an official publication of the American Association of Anatomists 2000, 219:287-303.

41. Varga ZM, Amores $A$, Lewis KE, et al: Zebrafish smoothened functions in ventral neural tube specification and axon tract formation. Development 2001, 128:3497-509.

42. Karlstrom RO, Trowe T, Klostermann $\mathrm{S}$, et al: Zebrafish mutations affecting retinotectal axon pathfinding. Development 1996, 123:427-38.

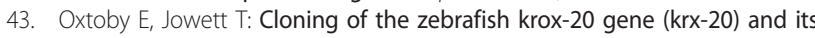
expression during hindbrain development. Nucleic Acids Research 1993, 21:1087-95.

doi:10.1186/1471-2164-13-2

Cite this article as: Milla et al:: Yeast-based assay identifies novel Shh/ Gli target genes in vertebrate development. BMC Genomics 2012 13:2.

\section{Submit your next manuscript to BioMed Central} and take full advantage of:

- Convenient online submission

- Thorough peer review

- No space constraints or color figure charges

- Immediate publication on acceptance

- Inclusion in PubMed, CAS, Scopus and Google Scholar

- Research which is freely available for redistribution

Submit your manuscript at www.biomedcentral.com/submit
Biomed Central 